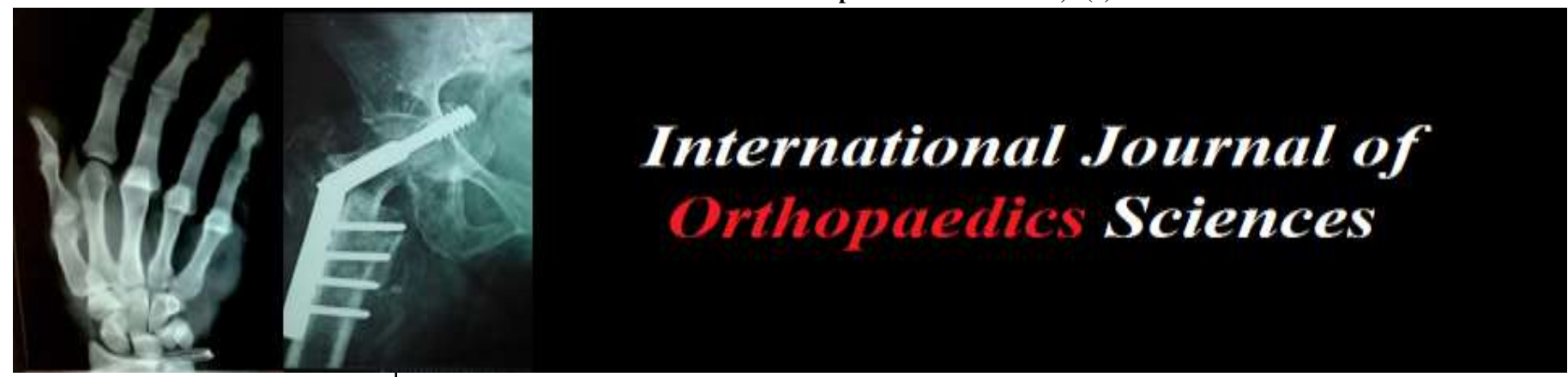

E-ISSN: 2395-1958

P-ISSN: 2706-6630

IJOS 2021; 7(4): 738-741

(C) 2021 IJOS

www.orthopaper.com

Received: 17-07-2021

Accepted: 06-09-2021

Dr. Mukul Kumar Singh

Senior Resident,

Department of Orthopaedics,

Dr. V M Government Medical

College \& Scsmsr, Solapur,

Maharashtra, India

Dr. Sunil P Handralmath

Professor \& HOD,

Department of Orthopaedics,

Dr. V M Government Medical

College \& Scsmsr, Solapur,

Maharashtra, India

Dr. Siddharth M Khadilkar

PG Resident,

Department of Orthopaedics,

Dr. V M Government Medical

College \& SCSMSR, Solapur,

Maharashtra, India

Dr. Nayan S Chaudhari

PG Resident,

Department of Orthopaedics,

Dr. V M Government Medical

College \& Scsmsr, Solapur,

Maharashtra, India
Corresponding Author:

Dr. Mukul Kumar Singh

Senior Resident,

Department of Orthopaedics,

Dr. V M Government Medical

College \& Scsmsr, Solapur,

Maharashtra, India

\section{Comparative study between functional outcome of high tibial osteotomy and partial fibulectomy in isolated medial compartment osteoarthritis of knee}

\author{
Dr. Mukul Kumar Singh, Dr. Sunil P Handralmath, Dr. Siddharth M \\ Khadilkar and Dr. Nayan S Chaudhari
}

DOI: $\underline{\text { https://doi.org/10.22271/ortho.2021.v7.i4k.2958 }}$

\begin{abstract}
Purpose: To compare the results of high tibial osteotomy with partial fibulectomy in the surgical management of isolated medial compartment osteoarthritis of knee and to reduce pain and deformity.

Methods: patients visiting the outpatient department of S.C.S.M.S.R. with complaints of knee pain. Patients who were found to have isolated medial compartment osteo-arthritis will be included in the study. 50 patients were subjected to high tibial osteotomy and 50 patients were operated with partial fibulectomy. Functional outcome of both the procedure were compared with WOMAC score. Patients were followed up at 3 months 6 months and 9 months and reassessed.

Result: There were a total of 100 patients with clinically and X-ray confirmed medial compartment osteoarthritis of knee (41 males and 59 females) with most patient in 51-60 year age group. mean pre operative WOMAC score was 82.38 and mean post operative score at 9 Month follow up was 38.43(p value $<0.001$ ) which is statistically significant. pre-operative VAS was 7.83 and post operative vas 2.78 and radiographic valgus angle preoperatively was 7.54 degrees of varus to postoperative valgus of $1.9^{0}$.

Conclusion: We want to conclude saying that partial fibulectomy is a safe, fast and affordable surgery to relieve pain and improve joint function and the medial joint space in human knee osteoarthritis. HTO is the surgery of choice in medial compartment OA associated with high degree of genu varum. From our study, it is quite clear that open wedge osteotomy using limb reconstruction system is an effective method of managing OA knee especially in younger patients with unicompartmental knee involvement. We strongly recommend this procedure in relatively younger patients as it may delay the need of joint replacement and in some cases, it may actually avoid it.
\end{abstract}

Keywords: High tibial osteotomy (HTO), partial fibulectomy, osteoarthritis (OA), Western Ontario and McMaster Osteoarthritis Index (WOMAC), (VAS) Visual analogue scale

\section{Introduction}

Osteoarthritis of the knee is often the cause of considerable disability, and pathological studies have shown that this joint is more commonly affected by degenerative changes than any other in the body ${ }^{[1]}$.

It is a common, debilitating disease which is associated with a large social and economic burden, in addition to the physical and psychological sequelae it often manifests in the affected individual. The OA of knee is more common in women, with female-to-male ratios varying between 1.5:1 and 4:1. Prevalence rates for knee OA, based on population studies in the US, are comparable to those in Europe. These studies report that severe radiographic changes affect $1 \%$ of people aged 25-34 and this figure increases to nearly $50 \%$ in those 75 years and above. Among participants aged over 45 years in the Framingham Study, the prevalence of radiographic knee OA was $19.2 \%$ and, in those over 80 years, the figure rose to $43.7 \%$ [3]. With the continued aging of the population and the alarming obesity epidemic, the prevalence of OA is expected to increase by $40 \%$ by $2025^{[4]}$.

Among the various surgical options joint replacement is quite popular as it gives long lasting pain relief besides good function. However, due to increase in life expectancy, reoperation may be required as no joint can last lifelong. Secondly in young high demand patients some activity restrictions may be required after arthroplasty. 
So herein lies the importance of High Tibial Osteotomy (HTO) which provides a well aligned, pain free and excellent functional knee for a pretty long time which may either delay a replacement for many years or may eliminate its need and Partial fibulectomy which is a lesser studied and lesser known procedure which is showing promising results in functional outcome and pain relief for isolated medial compartment osteoarthritis.

\section{Material and methods}

Our study is a longitudinal experimental study, single centered which includes Patients visiting the Outpatient department of Tertiary Care Centre in department of Orthopedics; with complaints of knee pain will be subjected to weight bearing x-ray of both knees ap/lateral. Patients who were found to have isolated medial compartment osteoarthritis were included in the study. 50 patients were subjected to high tibial osteotomy and 50 patients were operated with partial fibulectomy through randomized selection of patients. Functional outcome of both the procedure was compared with WOMAC score and VAS. Patients were followed up at 3 months 6 months and 9 months and reassessed.

Inclusion criteria: Patients in the age group of $>40$ years with moderate to severe symptomatic medial compartment OA of the knee, who are able to give informed consent for the surgery and Patients in whom conservative management has failed

Exclusion criteria: Posttraumatic knee OA, Inflammatory joint disease, Patients with a history of previous operations or fractures, Tricompartmental OA of knee.

\section{Detailed research plan}

Selection of patient: Patient above 40 yrs. of age irrespective of sex with Medial compartment primary osteoarthritis of knee Standing (weight bearing) X ray knee (anterio-posterior and lateral) is taken After admission of patient, preoperative WOMAC score and VAS was calculated, with informed written consent for procedure and after anaesthetic fitness patient was taken for operation. operative procedure included either resection of $2-3 \mathrm{~cm}$ of fibula, $6-10 \mathrm{~cm}$ from fibular head or High Tibial Osteotomy in which medial wedge osteotomy of proximal tibia is done with Limb Reconstruction System and post-operative WOMAC score and VAS was calculated. Patients were followed up on $3^{\text {rd }} 6^{\text {th }}$ and $9^{\text {th }}$ month and Analysis of results was done after significant statistical test.
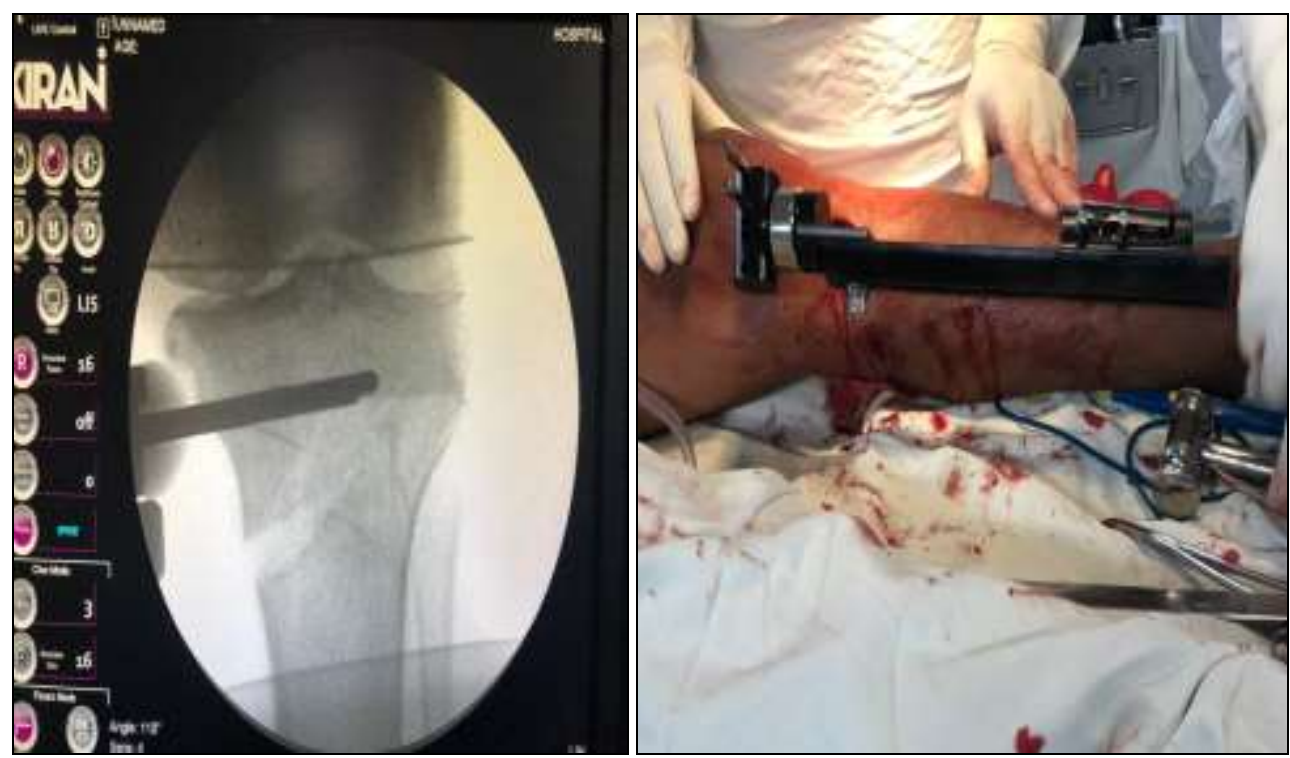

Fig 1; High tibial osteotomy with limb reconstruction system
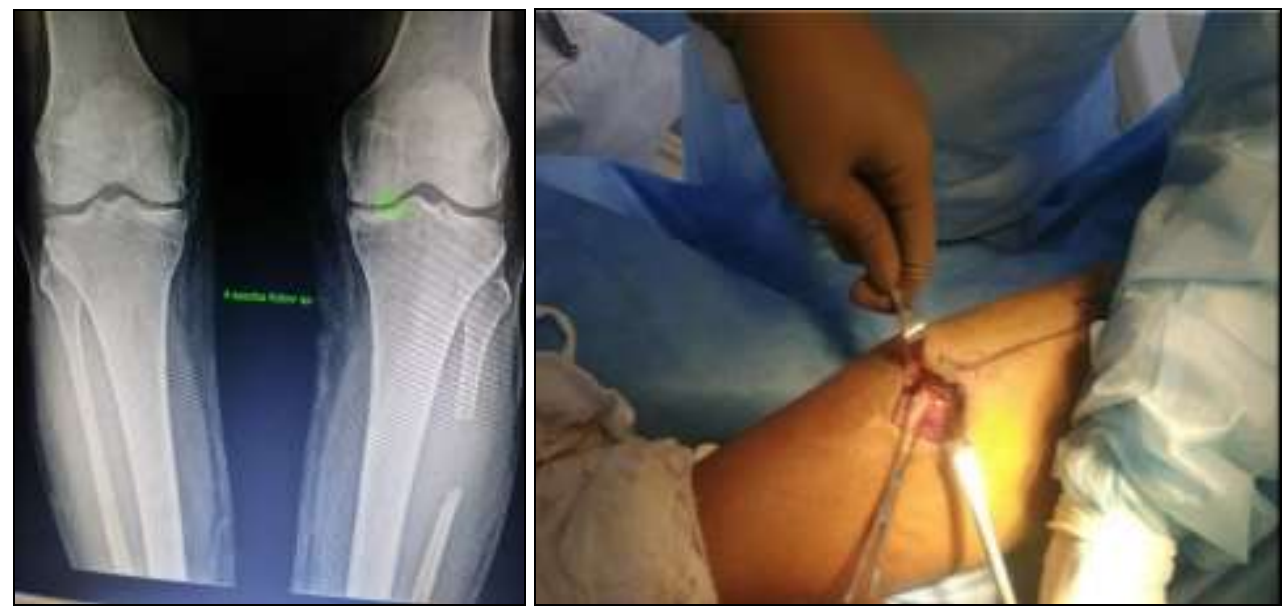

Fig 2: Partial Fibulectomy 


\section{Observations \& Results}

Table 1: Comparison between preoperative and postoperative assessment in high tibial osteotomy

\begin{tabular}{|c|c|c|c|c|c|c|}
\hline \multicolumn{2}{|c|}{ High tibial osteotomy } & Preoperative Assessment & Postoperative assessment & Test value* & P-value & Sig. \\
\hline \multirow{2}{*}{ VAS } & Mean \pm SD & $7.88 \pm 0.84$ & $2.78 \pm 1.28$ & \multirow{2}{*}{23.49} & \multirow{2}{*}{$<0.0001$} & \multirow{2}{*}{ HS } \\
\hline & Range & $7-9$ & $0-6$ & & & \\
\hline \multirow{2}{*}{ Womac } & Mean \pm SD & $82.56 \pm 5.344$ & $38.6 \pm 3.56$ & \multirow{2}{*}{48.40} & \multirow{2}{*}{$<0.0001$} & \multirow{2}{*}{ HS } \\
\hline & Range & $73-91$ & $33-47$ & & & \\
\hline \multirow{2}{*}{ Radiographic Angle } & Mean \pm SD & $7.52 \pm 3.28\left({ }^{\circ}\right.$ varus $)$ & $1.9 \pm 0.92\left({ }^{\circ}\right.$ valgus $)$ & & & \\
\hline & Range & $2-12$ & $1-4$ & & & \\
\hline
\end{tabular}

*: Paired t-test. The previous table (4) shows that there was highly statistically significant decrease in VAS at the postoperative assessment than preoperative assessment with p-value $<0.001$ while there was highly statistically decrease in WOMAC and radiological angle conversion from varus to valgus postoperative assessment than preoperative assessment with p-value $<0.001$ and $<0.001$ respectively

Table 2: Comparison between preoperative and postoperative assessment in partial fibulectomy

\begin{tabular}{|c|c|c|c|c|c|c|}
\hline \multicolumn{2}{|c|}{ Partial Fibulectomy } & $\begin{array}{c}\text { Preoperative } \\
\text { assessment }\end{array}$ & $\begin{array}{c}\text { Postoperative } \\
\text { assessment }\end{array}$ & $\begin{array}{c}\text { Test } \\
\text { value* }^{*}\end{array}$ & P-value & Sig. \\
\hline \multirow{2}{*}{ VAS } & \multirow{2}{*}{$\begin{array}{c}\text { Mean } \pm \text { SD } \\
\text { Range }\end{array}$} & $6.82 \pm 0.81$ & $2.82 \pm 0.81$ & \multirow{2}{*}{24.69} & \multirow{2}{*}{$<0.0001$} & \multirow{2}{*}{ HS } \\
\hline & & $6-8$ & $2-4$ & & & \\
\hline \multirow{2}{*}{ Womac } & \multirow{2}{*}{$\begin{array}{c}\text { Mean } \pm \text { SD } \\
\text { Range }\end{array}$} & $74.32 \pm 9.98$ & $34.6 \pm 6.58$ & \multirow{2}{*}{23.49} & \multirow{2}{*}{$<0.0001$} & \multirow{2}{*}{ HS } \\
\hline & & $60-90$ & $23-47$ & & & \\
\hline \multirow{2}{*}{$\begin{array}{c}\text { Radiographic } \\
\text { Angle }\end{array}$} & \multirow{2}{*}{$\begin{array}{c}\text { Mean } \pm \text { SD } \\
\text { Range }\end{array}$} & $5.94 \pm 2.31\left({ }^{\circ}\right.$ varus $)$ & $5 \pm 2.374\left({ }^{\circ}\right.$ varus $)$ & & & \\
\hline & & $2-9$ & $0-9$ & & & \\
\hline
\end{tabular}

The previous table(5) shows that there was highly statistically significant decrease in VAS at the postoperative assessment than preoperative assessment with p-value $<0.001$ while there was highly statistically significant decrease in WOMAC and radio postoperative assessment decrease in radiological varus angle than preoperative assessment with $\mathrm{p}$-value $<0.001$ and $<0.001$ respectively.

\section{Discussion}

Our study included 100 patients in department of orthopedics in a tertiary care center ( 41 males and 59 females), out of which 50 cases of $\mathrm{OA}$ knee were subjected to Partial Fibulectomy and 50 cases of OA Knee to HTO with the main aim to assess the efficacy in improving the knee pain and varus deformity.

Our study included 50 patients (32 female and 18 male) who were subjected to Partial Fibulectomy in patients of age group of 43 years to 77 years having mean ages of- 58.57 years. Functional outcome was measured using western Ontorio Macmaster University Score (WOMAC). Mean pre-operative WOMAC score was 73.65, mean post operative score at 9 months was 33.95. (p value <0.001) which is statistically significant and Mean VAS score preoperatively was 6.82 and post operatively was 2.82 which significant (p value $<0.001$ ). Complications include 2 superficial surgical site infections which healed subsequently and superficial peroneal nerve palsy in 1 patient recovered in 2 months with persistent paresthesia and tingling numbness in the distribution of superficial peroneal nerve. 1 deep peroneal nerve palsy recovered after a period of 6 months with persistent leg edema.

Remaining 50 patients in the age group of 45 years to 78 years having mean ages of- 62.35 years (27 female and 23 male) were subjected to HTO where in the Mean preoperative VAS score was 7.83, mean post operative score at 9 months was 2.78 . The mean pre operative womac score was 82.38 and mean post operative score at 9 Month follow up was 38.43(p value <0.001) which is statistically significant.

Females were the predominant sex. Obesity was the major risk factor in $\mathrm{OA}$ and strong association has been well established ${ }^{[5-7]}$. Preop varus deformity was 8-10 degrees in
$50 \%$ of cases. Our aim was to achieve postoperative mechanical axis of 6-8 degree valgus with Fujisawa point as reference. We could achieve 5-7 degree valgus in 60\% of the cases with high tibial osteotomy. Pain was the main criteria for performing this procedure and $100 \%$ of patients had moderate to severe pain preoperatively. However postoperative pain relief was pretty good with $70 \%$ patients having none or mild pain that too on strenuous exercise In this study, majority of the patients maintained or rather improved their preoperative range of motion. Only 4 patients had some decrease in range of motion postoperative (1520degree). Similar results have been described by Insall et al., ${ }^{[8]}$. So it is imperative to start knee mobilization as soon as possible.

In this study, majority of patients had radiological union at a mean of 12 weeks (10-16) after surgery. This observation coincides pretty well with study of Nakamura et al. ${ }^{[4]}$ In our studies complications include 3 cases of pin-tract infection and loosening and 3 patients of delayed union.

The overall outcome with this procedure was quite encouraging. With pain relief, range of motion and varus deformity correction as the major determining factors.

Functional outcome was measured using western Ontorio Macmaster University Score (WOMAC) The Mean follow up in our study was of 9 months

\section{Summary \& Conclusion}

Osteoarthritis of knee is a common debilitating disease of elderly, more common in females affecting daily activities of living. More common in Indian population because of squatting and sitting crossed leg habits. The osteoarthritis usually starts in medial compartment of knee progressing to involve anterior and lateral compartment.

Partial fibulectomy is a safe, fast and affordable surgery to relieve pain and improve joint function and the medial joint space in human knee osteoarthritis. It is Simple, Cost effective, Less morbid procedure.

The results showed all of the patients had significant reduction of pain compared with pre operative pain as assessed by VAS score.

Partial fibulectomy is a promising alternative in most 
developing countries because of their financial and healthcare delivery limitations. It may also constitute a promising alternative surgery for osteoarthritis of the medial compartment of the knee, especially for patients who cannot undergo Total Knee Arthoplasty because of certain medical comorbidities. Furthermore, these patients can still undergo TKA in the future if it becomes necessary.

HTO is the surgery of choice in medial compartment OA associated with high degree of genu varum

From the above study, it is quite clear that Open wedge Osteotomy using Limb Reconstruction System is an effective method of managing OA knee especially in younger patients with Unicompartmental Knee involvement. It is minimally invasive, requires no fibular osteotomy, minimal chances of nerve injury, easy to perform and no internal hardware used requiring subsequent removal. Besides correction is graduated and not one time as is seen with use of fixed angle plates where we may get under/over correction. We strongly recommend this procedure in relatively younger patients as it may delay the need of joint replacement and in some cases it may actually avoid it.

In short term follow-up Partial fibulectomy can be a better option in terms of Early mobilization, Preserving the normal bone stock around knee joint, Preserving the normal tibial alignment, Quick reproducible and will not hamper the future Total Knee arthroplasty, but in case of High Tibial Osteotomy along with significant improvement in pain \& functional outcome with correction of varus deformity of knee joint.

\section{References}

1. Jackson JP, Waugh W, Green JP, Harlow Wood: High Tibial Osteotomy For Osteoarthritis Of The Knee,The Journal Of Bone And Joint Surgery 1988.

2. Ashman RF, Ferguson PJ, IJdo JW, Lawry GV II. Rheumatic Diseases: Diagnosis and Management; Weinstein, Stuart L; Buckwalter, Joseph A; Turek's Orthopaedics: Principles and Their Application, 6th Edition: 2005 Lippincott Williams \& Wilkins 154-55.

3. Bijlsma JWJ. Strategies for the prevention and management of osteoarthritis of the hip and knee. Best Pract Res Clin Rheumatol 2007;21(1):59e76.

4. Woolf AD, Pfleger B. Burden of major musculoskeletal conditions. Bull World Health Organ 2003;81:646-56.

5. Coggon D, Reading I, Croft P, Mclaren M, Barrett D, Cooper C. Knee Osteoarthritis and obesity. Int J Obes Rel Met Dis. 2001;25(5):622-27.

6. Felson D, Zhang Y, Anthony J, Naimark A, Anderson J. Weight loss reduces the risk for symptomatic knee osteoarthritis in women: the Framingham study. Ann Intern Med. 1992;116(7):535-39.

7. Felson D, Anderson J, Naimark A, Walker AM, Meenan RF. Obesity and knee osteoarthritis: the Framingham study. Ann Intern Med. 1988;109(1):18-24.

8. Insall JN, Shoji H, Mayer V. High tibial osteotomy. A Five Year evaluation. J Bone Joint Surg 1974;56:1397405.

9. Nakamura E, Mizuta H, Takagi SK, Sakamato K. Open wedge osteotomy of the proximal tibia with haemicallotasis. J Bone Joint Surg Br 2001;83(8):111115 . 